\title{
Prävention aus interdisziplinärer Sicht - Überlegungen zum Fall Hamburg
}

Heike Herrmann

\section{Einleitung}

A rchitektur und bebaute Umwelt nehmen Einfluss auf die "Sicherheit in der Stadt» (vgl. Kodolitisch 2003: 5f) - diese Erkenntnis hat sich insbesondere seit der letzten Jahrhundertwende sowohl in der Stadtplanung als auch in der Kriminologie durchgesetzt. Wie dies jedoch geschieht, ist nach wie vor ein Rätsel. Der Ruf der Praktiker vor Ort, aber auch von Politik und Planung nach Richtlinien, an denen sich die Kommunen orientieren könnten, hat inzwischen zu ersten Entwicklungsschritten einer EU-Norm geführt. Sie soll längerfristig zu einer sicheren städtebaulichen Umwelt führen (vgl. van Soomeren/Mölk 2001; Schubert / Schnittger 2002: 15; Weicht 2003). Sicherheit hat dabei immer zwei Dimensionen: Zum einen geht es um eine Verringerung von Kriminalität, also um Kriminalprävention im engeren Sinne. Zum anderen sollen Maßnahmen (unabhängig von der objektiven Sicherheitslage) das subjektive Sicherheitsgefühl erhöhen. Ein Element des Sicherheitsgefühls ist dabei die Kriminalitätsfurcht. Sie wird nicht unerheblich von Anzeichen von ,Disorder' (Skogan 1990) und anderen Faktoren des räumlichen Kontextes sowie von der subjektiven Problembelastung der Bewohner bestimmt (vgl. Hermann/Laue 2005: 207). Mit diesen beiden Gruppen von Einflussfaktoren auf die Sicherheit in der Stadt und Maßnahmen zur Verbesserung des Sicherheitsgefühls in der Stadt Hamburg wird sich der folgende Beitrag beschäftigen.

Ein Blick in die internationalen Erfahrungsberichte zeigt, dass Deutschland bezüglich der Entwicklung sozial-räumlicher Konzepte der kommunalen Kriminalprävention immer noch im Hintertreffen zu sein scheint. Dies gilt besonders für Hamburg: Während aus den anderen Bundesländern bereits zahlreiche Berichte vorliegen, existiert in der Hansestadt bis heute kein Landespräventionsrat oder ein ähnliches Organ, das den Sachstand gesamtstädtisch wiedergeben könnte (vgl. Steffen 2005: 156). ${ }^{1}$ Dies verwundert, denn gerade in dieser Stadt war das Thema "Sicherheit « in den letzten Jahren ein wichtiges Element des politischen Diskurses. Die ,Partei der Rechtsstaatlichen Offensive' hatte mit diesem Thema im September 2001 die jahrzehntelange sozialdemokratische Regierung zu Fall gebracht.
Der folgende Beitrag soll - ohne den Anspruch auf Vollständigkeit zu erheben - einen knappen Einblick in die kriminalpräventiven Maßnahmen der Stadt bzw. des Landes Hamburg geben. ${ }^{2}$ Die Schilderung der Stadtteilentwicklung einer Hamburger Großwohnsiedlung aus den 70er Jahren vermittelt anschließend einen gebietsspezifischen Einblick in die Hamburger Praxis. Es handelt sich um einen Raum- und Gebäudetyp, der gemeinhin zahlreiche Tatgelegenheitsstrukturen, Kleinkriminalität, soziale Probleme und Ängste zu bieten hat. Die aus der Sicht von Experten geschilderte Entwicklung der Großwohnsiedlung verbindet über die Praxis vor Ort Prinzipien und Vorgehensweisen der ,sozialen Stadtentwicklung' mit denen der kommunalen Kriminalprävention. Es wird deutlich, dass sich kriminalpräventive und stadtsoziologische Ansätze der Gebietsentwicklung zu einem neuen, interdisziplinären Präventionsverständnis zusammenführen lassen. Dieses könnte dazu führen - mehr als es im Rahmen der klassischen Kriminalprävention der Fall ist - der Verminderung von Kriminalitätsfurcht und allgemeiner Unsicherheiten (der »zweiten Zieldimension von Kriminalprävention «, Heinz 2005: 12) ein gutes Stück näher zu kommen. Aus dieser Perspektive ist Hamburg mit seinen präventiven Maßnahmen nicht ganz so weit von anderen Bundesländern entfernt, wie es zunächst scheinen mag.

Der Beitrag stützt sich auf die Ergebnisse des im 5. Rahmenprogramm der Europäischen Union finanzierten internationalen Forschungsprojekts »Insecurity in European Cities « $(\mathrm{InSec})^{3}$ : In fünf europäischen Städten (Amsterdam, Budapest, Hamburg, Krakau und Wien) wurden eine Expertenbefragung ${ }^{4}$ sowie eine repräsentative schriftliche Befragung und Tiefeninterviews mit Bewohnern zweier Untersuchungsgebiete durchgeführt. Die als Beispiel gewählte Großwohnsiedlung ,Steilshoop', ergänzt um einen kleinen Teil eines angrenzenden Stadtteils, war eines der Hamburger Untersuchungsgebiete (zum Aufbau des Projektes vgl. Herrmann/Sessar/Weinrich 2003). Für die Hamburger Untersuchungsgebiete liegen zusätzlich Beobachtungsprotokolle vor (vgl. Flick 1995: 252ff; Brüsemeister 2000: 82ff), die weitere Auskünfte über das Leben in den Gebieten geben. Der Beitrag schließt zudem an das erste Heft dieses Jahres an, in dem Wolfgang Stangl und Janina Czapska auf die unterschiedlichen Bedeutungen von Kriminalprävention in den Städten Wien und Krakau hingewiesen haben. Der in dem gemeinsamen Forschungsprojekt intensiv geführte fächerübergreifende Diskurs zwischen Kriminologie und Stadtsoziologie sowie -planung findet hier eine Fortsetzung.

\section{Prävention in Hamburg ${ }^{5}$}

Zentrale Schwerpunkte der repressiven und präventiven Polizeiarbeit der Stadt Hamburg liegen nach Aussagen der Experten in den Bereichen der Drogenbekämpfung sowie der Bekämpfung und Verhütung von Kinder- und Jugendkriminalität. ${ }^{6}$ Mit Bezug auf die im Lebensverlauf von Jugendlichen auftretende Gelegenheitskriminalität gestaltete sich der Umgang mit jugendlichen Ersttätern noch in den 90er Jahren im Allgemeinen so, dass niedrigschwellige staatliche Reaktionen bei Bagatellkriminalität bevorzugt wurden. Dies bedeutete zunächst eine schriftliche Ermahnung des Jugendrichters und bei Wiederholungsfällen ein Ermahnungsgespräch, ggf. mit der Anregung eines Täter-Opfer-Ausgleichs. Informelle erzieherische Maßnahmen (wie Arbeitsleistungen in sozialen Einrichtungen) wurden der formellen Bestrafung vorgezogen. Noch im Jahr 2000 bewertete eine eigens zur Überprüfung dieser Umgangsweise durch die Bürgerschaft eingesetzte Kommission diese Praxis als positiv (vgl. Kastner / Sessar 2001). Nach dem Regierungswechsel im Jahr 2001 wurde hingegen ein schärferes Vorgehen gegen die jugendlichen Straftäter verabredet. Zusätzlich wurde die geschlossene Heimunterbringung wieder eingeführt.

Im Mittelpunkt präventiver Maßnahmen steht in Hamburg wie in anderen Großstädten häufig die Schule. In Hamburg hatte eine zwischen dem Amt für Schule, dem Amt für Berufliche Bildung und Weiterbildung und der Polizei vereinbarte Kooperation (genannt »Cop4U«, übersetzt: Ein Polizist für dich) das Ziel, über eine vertrauensvolle Zusammenarbeit zwischen den Schulen und der Polizei gemeinsame Maßnahmen zur Eindämmung der Jugendkriminalität zu verabreden und umzusetzen. Dies bedeutet in der Praxis, dass die eingesetzten ,Bürgernahen Beamten (ähnlich den Kontaktbeamten in Wien; Stangl 2005: 10) zum Teil ausschließlich für die Schulen zuständig sind. In der Schule - wie im Wohngebiet - ermöglichen die ,Bürgernahen Beamten' eine leichtere Kontaktaufnahme, beraten, informieren über polizeiliche Reaktionen auf aktuelle Vorfälle und regen alle Beteiligten an, erlittene 
oder beobachtete Straftaten zu melden. Sie sind über ihre Präsenz in den Gebieten ein wesentliches Element der präventiven Arbeit in den Stadtteilen. Zusätzlich startete im Jahr 2002 ein mit den Schulen verabredetes Präventionsprogramm »Kinder- und Jugenddelinquenz«: ausgewählte Polizeibeamte geben in den Schulen Präventionsunterricht (2002 3.078, 20033.790 Unterrichtseinheiten).

Seit 2003 meldet die Jugendbeauftragten der Polizei zudem aus ihrer Sicht besonders gefährdete Minderjährige an das in der Behörde für Soziales und Familie gebildete Familieninterventionsteam (FIT) zum Zwecke weiterer Maßnahmen. Schließlich wurde eine Aktion »Rücksicht auf Kinder...kommt an « gestartet. Kooperationspartner waren neben der Polizei die Behörde (= Ministerium) für Bildung und Sport, die Verkehrswacht, der ADAC, die Landesunfallkasse und die Hamburger Elternkammer. Ziel war es, durch Verkehrskontrollen, Informationsveranstaltungen und Beratungsgespräche auf ein verändertes, auf die spezifische Gefährdung von Kindern achtendes Verhalten im Straßenverkehr hinzuwirken.

Erwähnt seien an dieser Stelle außerdem:

a, die Aktion ,Findelbaby', die es ungewollten Müttern u. a. ermöglichte, ihr neugeborenes Kind in einer Babyklappe anonym abzugeben. Das Projekt bewirkte, dass im Jahr 2001 keine Babys mehr ausgesetzt wurden;

b, die Aktion ,Gemeinsam gegen Gewalt', die sich mit der Gewalt und dem Raub unter Jugendlichen beschäftigt. Die Hamburger Polizei führte hier gemeinsam mit dem Referat Gewaltprävention des Amtes für Schule, dem Jugendinformationszentrum Hamburg (JIZ) sowie dem Fachkreis Gewaltprävention eine Kampagne durch, die sich vor allem auf die gewaltsame Wegnahme von Handys bezieht;

c, die Kampagne, Wer nichts tut, macht mit', die an Zivilcourage und Hilfsbereitschaft appelliert sowie

d, die immer wieder auf Zeit und zu lokalen Problemen gegründeten ,Sicherheitspartnerschaften' zwischen Bürgern und Polizei, die über die Erhöhung der objektiven und subjektiven Sicherheit der Bürger die Lebensqualität erhöhen sollen.

Alle genannten Aktionen und Projekte sind im ,Infopool Prävention' des Bundeskriminalamtes verzeichnet. Gebiete, in denen diese und ähnliche Projekte primär durchgeführt werden, sind häufig die Gebiete, die sich durch sehr komplexe Problemlagen und, damit verbunden, eine hohe Gewaltbereitschaft in der Bevölkerung auszeichnen. Ein in der Vergangenheit so zu charakterisierendes Gebiet war die Großwohnsiedlung Steilshoop, die im Folgenden als Fallbeispiel dient.

\section{Ein Beispiel interdisziplinärer Prävention: Die Großwohnsiedlung Hamburg Steilshoop}

Nicht zufällig wird an dieser Stelle als Beispiel eine Großwohnsiedlung ausgewählt: Neben den innerstädtischen Altbaugebieten sind sie es, die sowohl in stadtsoziologischen als auch in kriminologischen Forschungen neben sanierungsbedürftigen Altstadtquartieren im Fokus der Betrachtung stehen (vgl. z. B. Häußermann 2000: 14; Schubert / Schnittger 2002; Oberwittler 2003: 43; Mayer 2004: 9). Sollen zudem baulich-räumliche Aspekte in die Konzeption der Prävention einfließen, so muss sich die Betrachtung und Analyse auf einen bestimmten Raum-Typus beziehen. Hochhäuser und ihre Umgebung bedürfen einer anderen städtebaulichen Entwicklung als dies bei Gründerzeitquartieren der Fall ist. In ihnen bestimmt eine gänzlich andere architektonische Gleichförmigkeit das städtische Leben und werden ganz andere Gruppengrößen erreicht, wenn es etwa um die Beteiligung von Bewohnern geht. Diese Unterschiede sind zu berücksichtigen. So sind die präsentierten Ergebnisse über den spezifischen Raum-Typus auf andere Großwohnsiedlungen und Städte übertragbar. Die in der städtebaulichen und sozialen Dimension verfolgten Prinzipien, wie die Beteiligung der Bewohner und die Schaffung von Verantwortungsbereichen, lassen sich dagegen auch in anderen Raum-Typen mit ähnlichen Problemen verfolgen.

\section{Die ersten Jahre der Siedlung}

Steilshoop ist mit über 6.000 Wohneinheiten die zweitgrößte Großwohnsiedlung Hamburgs (Harms / Schubert 1988: 403); der Stadtteil hatte im Jahr unserer Untersuchung 2003 laut statistischem Landesamt 19.516 Bewohner. ${ }^{7}$

Der Beginn der Geschichte der Großwohnsiedlung ,Neu-Steilshoop' (im Folgenden weiter: ,Steilshoop') ist der Anfang eines großen städtebaulichen und damit auch sozialen Experiments: Es sollten die »Grundsätze für Demonstrativbauvorhaben « beachtet werden (Beyerlein 1980 mit Bezug auf Schäfer 1979: 54), indem etwa die Bildung kleinräumiger Nachbarschaften städtebaulich und sozialpädagogisch unterstützt wurde (z. B. durch die Planung von Gemeinschaftsräumen und Innenbereichen oder etwa auch den Betrieb von Jugendwohnungen, die Jugendliche aus Problemfamilien vor dem Heim bewahrten). Auch sollten die Wohnungen selbst einem qualitativ hochwertigen Standard genügen. Nach einer langjährigen Planungsphase begann die Bebauung am Ende der 60er Jahre und wurde bis in die 70er Jahre fortgesetzt. Im Ergebnis erreichten die ,Wohngebirge' in der Mitte der Siedlung bis zu 17 Geschosse. ,Ringe' sollten die Grundlage größerer, einzelne Blöcke mit Gemeinschaftsräumen, die Grundlage kleinerer Nachbarschaften sein. Sehr unterschiedliche Investoren brachten eine heterogene Eigentümerstruktur und eine Konzentrationen des sozialen Wohnungsbaus innerhalb der Siedlung direkt neben Bereichen mit Eigentumswohnungen hervor.

Bereits in den 80er Jahren mussten im »Wohnmodell Steilshoop« (Beyerlein 1980) Nachbesserungen vorgenommen werden. Die soziale Infrastruktur erwies sich trotz allem Bemühen als mangelhaft; so waren insbesondere die Kinder unter 12 Jahren nicht ausreichend versorgt (vgl. Meier 1985: 226f). Vor allem in den hohen Häusern der Mittelachse (»übelster, scheußlichster Präfabrikationswohnungsbau aus der Tube «, so ein Architekt 1975, zitiert in Meier 1985: 230) war es zu schweren Konflikten zwischen den Bewohnern, häufigen Krisen und Polizeieinsätzen gekommen: "In der Anfangsphase, nachdem Steilshoop gebaut wurde, gab es Probleme, weil es ein Stadtteil war, der mit Sozialwohnung durchsetzt war und die entsprechende Klientel da eingezogen ist. Und wer so dicht aufeinander wohnt, da gibt es natürlich Konfliktpotential. Und da gab es von Kfz-Delikten über Körperverletzung, Brandstiftung und dergleichen sehr viel« (EX PK 2001: 2) ${ }^{8}$. Nach dem Erstbezug durch die Kriegsgeschädigten kamen nun in einer zweiten Welle Mietparteien über Zwangszuweisungen in die Sozialwohnungen des Gebiets. Neben der auftretenden Gewaltkriminalität entwickelte sich dennoch der Leerstand zu einem Problem. Manche Vermieter ließen ihre Wohnungen lieber leer stehen, als sie durch unbeliebte Mieter »verwohnen« zu lassen. Gleichzeitig dramatisierte sich die Situation innerhalb der Häuser des sozialen Wohnungsbaus. Anfang 1985 standen schließlich ca. 200 Wohnungen leer. Leerstand bedeutete vor allem einen Verlust an sozialer Kontrolle, ist aber auch - in Form der gardinenlosen Fenster - ein Zeichen dafür, dass es sich an diesem Ort nicht gut leben lässt (vgl. Flade 2003). Es entstand eine Situation, die sowohl in der Stadtsoziologie als auch in kriminologischen Beobachtungen so häufig beschrieben wurde: Durch den Wegzug derer, die sich ein attraktiveres Wohnumfeld leisten können, wird die räumliche Konzentration finanziell schwacher Haushalte weiter verstärkt. Die Kaufkraft im Gebiet wird zusätzlich geschwächt (vgl. Herrmann 2000: 215f; Becker / Franke / Löhr / Schuleri-Hartje 2003: 11). In der ökonomischen Dimension setzt ein Kreislauf von sinkender Nachfrage in bestimmten Segmenten (z. B. im Bezug auf Kleidung) und weiter sinkendem Angebot ein. In der kulturellen Dimension kommt es zur Stigmatisierung des Gebiets und zur Diskriminierung der Bewohner.

Die Situation im Stadtteil wies am Ende der 80er Jahre schließlich alle Charakteristika auf, die Gewaltkriminalität wahrscheinlich werden lassen und als Furcht auslösend gelten (in Anlehnung an Kube 2003; Hermann / Laue 2001): ${ }^{9}$ 
- niedriger sozialer Status der Bewohner verbunden mit schlechten Zukunftschancen vor allem der Kinder und Jugendlichen (ein hoher Bestand an Sozialwohnungen),

- Konzentration benachteiligter Haushaltsformen (kinderreiche Familien, allein erziehende Elternteile),

- Konzentration ethnischer Minderheiten,

- Stigmatisierung des Stadtteils von, außen' (unterstützt durch die lokalen Printmedien),

- günstige Tatgelegenheitsstrukturen aufgrund hoher Anonymität,

- hohe Dichte von Zielobjekten kriminellen Verhaltens (z. B. Kinder und Jugendliche, ältere Menschen)

- hoher Anteil männlicher jugendlicher Gelegenheitstäter, ${ }^{10}$

- in Teilen defizitäres, monofunktionales Umfeld (mangelnde Möglichkeiten des ungeplanten, unverbindlichen Zusammenseins und der Kommunikation),

- hohe Wohndichte ${ }^{11}$ und wenig Raum zur individuellen Entfaltung insbesondere für die Kinder und Jugendlichen des Gebiets.

»Da praktisch nur noch über das Wohnungsamt neue Bewohner in leerstehende Wohnungen eingewiesen werden konnten, die aber die soziale Labilität jeweils noch erhöhten, wurde seitens der betroffenen Vermieter Initiative ergriffen « (Harms / Schubert 1988: 226). Die Wohnungsgesellschaften und vor allem die rechtlich nicht so stark an die Wohnungszuweisungen gebundenen Eigentümer-Genossenschaften

a, versuchten über gezielte Maßnahmen das Image der Siedlung zu verbessern,

b, griffen die Problemhochhäuser heraus und vermieteten hier nur noch an ausgewählte Personengruppen und

c, setzten Sozialarbeiter/innen zur Betreuung der Mieter ein.

Auch städtebaulich wurde der Versuch unternommen, die Qualität der Siedlung zu erhöhen:

a, Hauseingänge wurden freundlicher gestaltet, $\mathrm{b}$, erste Fassaden gestrichen und

c, mehr Grün angelegt.

»Die Wohnungsbaugesellschaften haben die Leute mit niedrigen Mieten bzw. zum Teil mit drei Monaten Freimiete gelockt, sie haben dort an den Häusern etwas gemacht. Die Gartenbauämter haben da Bäume gepflanzt und mehr Bänke hin gestellt. Das Einkaufszentrum wurde teilweise erneuert und erweitert. Die entscheidenden Leute an diesen Runden Tischen haben auch zusammen gearbeitet, während man früher gegeneinander gearbeitet hat. Als man die Dinge dann miteinander abgestimmt hatte, fußten und fassten diese Maßnahmen dann auch entsprechend (EX PK 2001: 3).
Zudem leitete die zuständige Behörde Maßnahmen ein, um die Siedlung als Sanierungsgebiet zu erklären und damit Zuschüsse zur Verbesserung der Bausubstanz und des Wohnumfeldes gewähren zu können. Es wurde der Versuch unternommen, sowohl die baulich-physische Seite der Siedlung zu verbessern als auch notwendige soziale Unterstützung zu leisten. »Ursprünglich war das ja mal ein belasteter Stadtteil. Es kommt ja nicht von ungefähr, dass Steilshoop so einen schlechten Ruf hat, das liegt ja nicht nur an den Hochhäusern, sondern auch daran, was da passiert ist. Aber man hat dort versucht, das Beste daraus zu machen - auch mit einem sehr hohen finanziellen Aufwand « (EX PK 2001: 2f). Möglichst viele der Bereiche, die erfahrungsgemäß nur ungern abends oder in der Nacht betreten werden (wie die Parkgaragen oder gering beleuchtete Grünbereiche) sollten anders genutzt und hierdurch verändert werden. Zusätzlich wurden die Bemühungen verstärkt, auftretendem Vandalismus oder auch den Graffiti entgegen zu treten, indem Jugendliche in die Gestaltung eingebunden und Schäden (entsprechend dem »broken windows «-Ansatz; vgl. Hess 1999) so schnell wie möglich beseitigt wurden.

Zusätzlich wurden über Sanierungs- bzw. Revitalisierungsvorhaben ${ }^{12}$

- Kommunikationsräume (attraktive Spielplätze, Grünflächen und Fußwege) und

- Verantwortungsbereiche (z. B. über Mietergärten, aber auch Attraktivitätssteigerung von Balkonen) im direkten Wohnumfeld geschaffen.

- Hierdurch wurde gleichzeitig die soziale Kontrolle erhöht (Beobachtung des öffentlichen Raumes).

Über die gemeinsam organisierte Feste und Veranstaltungen sowie neue Infrastruktureinrichtungen für Jugendliche, Alleinerziehende, ältere Menschen und Gruppen unterschiedlicher Kulturen wurde die Anonymität verringert und die Hemmschwelle gesenkt, sich mit den Nachbarn auseinander zu setzen oder auch Hilfe von offizieller Seite einzufordern.

Soziale Einrichtungen insbesondere für Kinder und Jugendliche beteiligen sich, ebenso wie die Kirchen und Vertreter der Schulen, der politischen Parteien, der Polizei und des Einzelhandels "alle die, die an der Gestaltung in irgendeiner Form mitwirken« (EX PK 2001: 6) - an Arbeitskreisen und Runden Tischen, die auch heute noch als Frühwarnsystem funktionieren: „So kommen die Verantwortlichen auf die Kollegen vor Ort oder auch auf uns zu und berichten, wo sich was zusammen braut. Selbst von Kleinigkeiten bekommen wir so Kenntnis und können die sofort im Keim ersticken. Das bewirken diese Runden Tische eben auch, wenn nichts zu besprechen ist « (EX PK 2001: 15).
Durch Aktivitäten, von unterschiedlichen Akteuren im Stadtteil initiiert (Müllsammelaktion, Sport, Feste usw.), bleiben die Arbeitskreise informiert, was den Stadtteil bewegt, welche Probleme aktuell und welche Potentiale derzeit aktiv sind. Neben der Verbesserung der Lebensqualität im Stadtteil sind die Aktivitäten somit ein wichtiges Element des Frühwarnsystems.

Allen hier aufgeführten Maßnahmen ist gemeinsam, dass sie nicht in der ganzen Siedlung und nicht immer in gleichem Maße durchgeführt wurden. So verweist die ,Sozialraumanalyse des Jahres 2000 für das Gebiet Neu-Steilshoop immer noch auf einen Mangel in der kulturellen und sozialen Infrastruktur des Stadtteils hin, hebt jedoch gleichzeitig den "teilweise dörflichen Charakter« der Siedlung mit funktionierenden multikulturellen Kommunikationsstrukturen hervor (Baisch-Weber 2000: 44f). In einigen Bereichen wurden die Verbesserungen erst sehr spät eingeleitet: Zum Zeitpunkt unserer Untersuchung, rund 20 Jahre nach dem Beginn der ersten Maßnahmen, waren in einigen Blocks weder die Schließanlagen noch die Fenster modernisiert. Insgesamt wurde die Lebensqualität der gesamten Großwohnsiedlung jedoch durch die Bemühungen einer Vielzahl von Akteuren erheblich erhöht.

\section{Zur heutigen Situation}

Durchgeführte Beobachtungen in der Siedlung vermitteln heute ein sehr ,aufgeräumtes', wenn auch nach wie vor in den Größendimensionen gigantisches Bild einer Hochhaussiedlung. Die wechselhafte Entstehungsgeschichte hat dazu geführt, dass Steilshoop von sehr unterschiedlichen Baukonzepten und architektonischen Bildern geprägt ist. Die gesamte Blockstruktur mit den geschlossenen Straßen- und Hofräumen lässt jedoch trotzdem ein Ganzes entstehen und ist Charakteristikum der Siedlung (s. Abb. 1).

Bis zu vierspurige Zufahrtsstraßen sowie einer Vielzahl von Parkpaletten und -garagen sind ein weiteres bleibendes Kennzeichen der (autogerechten) Siedlungsstruktur. Einen direkten, nicht über den Busverkehr vermittelten Anschluss an das öffentliche Nahverkehrsnetz hat die Großwohnsiedlung bis heute nicht bekommen, obwohl immer wieder in der Öffentlichkeit und Politik hierüber diskutiert wurde. So genannte »Pufferzonen« (Kleingärten, Gewerbegebiete und Grünflächen) trennen die Großwohnsiedlung vom Rest der Stadt und führen dazu, dass kaum ein Nicht-Steilshooper die Siedlung betritt; im Hinblick auf die »isolierte Lage « (Beyerlein 1980: 25) Neu-Steilshoops hat sich nichts verändert. Dies sind Strukturen, die unverrückbar bestehen blieben.

Das angelegte viele Grün in und um die Siedlung ist inzwischen jedoch gleichzeitig ein Kenn- 
zeichen hoher Attraktivität geworden, denn es ermöglicht ein relativ naturnahes innenstadtnahes Wohnen.

Die Zuzüge und Fortzüge zeigen an, dass mehr Menschen in den Stadtteil ziehen als dass sie ihn verlassen.

Der Anteil der Ausländer/innen liegt mit 17,7\% im Hamburger Gesamtvergleich weiterhin relativ hoch, bei geringfügig steigender Tendenz. Unter Berücksichtigung der Tatsachen, dass

a, eine große, nach 1999 verstärkt zuwandernde Gruppe (die als Russlanddeutsche bezeichneten Bewohner der ehem. Sowjetunion) nicht als Ausländer registriert werden, sondern die deutsche Staatsangehörigkeit erhalten und

b, seit Mitte der 90er Jahre bis zum Beginn des 21. Jahrhunderts eine Einbürgerungswelle insbesondere türkischer Bewohner zu verzeichnen war,

ist von einem relativ hohen Anteil an Bewohnern mit Migrationshintergrund auszugehen. ${ }^{13}$ Der hohe Anteil ethnischer Minderheiten vermittelt - anders als in anderen Gebieten Hamburgs - ein sehr heterogenes Bild von Kulturen, da im Straßenbild von Steilshoop keine Minderheitengruppe dominiert. Die am Ende der 70er Jahre in die Siedlung gezogenen Migranten aus den Hauptanwerbeländern Türkei, Jugoslawien, Portugal, Griechenland, Italien und Spanien (vgl. Meier 1985: 216), damalige Aussiedler aus Polen wie Flüchtlinge und Zuziehende aus den afrikanischen und arabischen Staaten wohnen auch heute noch in der Großwohnsiedlung.

\section{Abb. 1: Bilderbuchrasen mit Trollfiguren}

\section{Bilderbuchrasen mit Trollfiguren Steilshoop: Blumen, Windmühlen und Zwerge zieren die Pazelle der Tabels.}

Von Claudia Sewig

Die braune Holzgartenpforte von Parzelle 23 schwingt gut geölt auf. In der Laube klingelt es daraufhin im Dreiklang: ein Bewegungsmelder kündigt Besucher an. Herbert Tabel (80) kommt von der Terrasse um die Ecke, freut sich über die erstaunten Blicke: „Habe ich selbst eingebaut!“ Und bittet in seine 350 Quadratmeter große, ordentliche Gartenwelt in der Kolonie der Gartenfreunde an der Seebek.

Hier, in Steilshoop, pflegen Herr Tabel und seine Frau Ruth (72) seit 23 Jahren Dahlien, Astern und Fleißige Lieschen, eine kleine Auswahl an Zwergen - und einen Bilderbuchrasen.

Г... 1

Quelle: Hamburger Abendblatt vom 16. 09. 2003
Bestände wiederum nur in bestimmten Bereichen der Siedlung zu finden, so dass sich die innere Segregation bzw. die Konzentration finanziell schwacher Mieter in Teilgebieten der Siedlung zum Beginn des 21. Jahrhunderts wiederum verschärft.

Trotz der sich verschärfenden subjektiven Problembelastung der Bewohner und seit dem Jahr 2002 anhaltender Kürzungen im Bereich der sozialen Infrastruktur fanden wir im Rahmen unserer Forschung nur sehr wenig vor, was für die Existenz von hohen Verunsicherungen oder ,Angsträumen' sprechen könnte. Die Einschätzung der Atmosphäre im Stadtteil ergab, dass sich lediglich $10 \%$ der Befragten dieses Untersuchungsgebiets ${ }^{16}$ unsicher fühlen. $45 \%$ bezeichneten den Stadtteil als ,vertraut' und $66 \%$ als ,ruhig' bzw. ,sehr ruhig'. Lediglich bei den Skalen ,attraktiv unattraktiv' und ,schmutzig - sauber' lagen die Antworten im Mittelfeld, alle anderen Beurteilung waren positiv.

Auf die Nachbarn ist im Notfall Verlass, darüber hinaus bestehen wenig Kontakte. Die qualitative Befragung hat jedoch gezeigt, dass relativ häufig enge verwandtschaftliche Beziehungen im Stadtteil und damit in der weiteren Nachbarschaft bestehen: Gemeinsame Einkäufe mit dem Auto in entfernten Subzentren der Stadt, gemeinsames Essen usw. sind Kennzeichen dieser Beziehungen. So ist es auch kein Problem, den einzigen Ort, an dem sich die Bewohner »unwohl « fühlen, die Mittelachse der Siedlung mit dem Schulund Einkaufszentrum, zu meiden. Hier ist es auch weniger die Angst vor Übergriffen, als vielmehr die mangelnde Attraktivität und Gelegenheit, die zur Vermeidung führt. Wurde bei den Großeinkäufen etwas vergessen, so wird der tägliche Bedarf auch im Supermarkt des Stadtteilzentrums gedeckt. Für einige ältere Menschen ist das Einkaufszentrum zudem der Ort, an dem sie kommunizieren und lose Kontakte pflegen.

Sparmaßnahmen in den Bereichen der sozialen und kulturellen Infrastruktur (Cafés und andere Stadtteiltreffpunkte, deren Personal mit öffentlichen Mitteln finanziert wurde, Sportvereine u.v.a.; s. Artikel des Hamburger Abendblattes) führen jedoch immer wieder, wenn auch nicht unmittelbar zur Schließung, so doch zur permanenten Unsicherheit in den fest installierten Strukturen der Teilnahme der verschiedensten Gruppen am öffentlichen Leben. ${ }^{17}$ entlassen worden und stehen nun dem freien Wohnungsmarkt zur Verfügung. ${ }^{15}$ Möglichkeiten der Stabilisierung von Nachbarschaften durch entsprechende Mieter ergeben sich jedoch nur dann, wenn dieses Wohnraumangebot auch nachgefragt wird. Zudem sind diese
Insgesamt ist die Situation im Stadtteil zum Zeitpunkt unserer Untersuchung trotz sich insgesamt verschlechternder gesellschaftlicher Rahmenbedingungen stabil. Es zeichnet sich jedoch $\mathrm{ab}$, dass die Zukunft ein verändertes Agieren sowohl der Akteure vor Ort (Wohnungsgesellschaften und andere wirtschaftliche Akteure, soziale Infrastruktur, Polizei und Bewohner) als auch der städtischen Politik erfordert. 


\section{Zur Rolle der Politik}

Von der Sanierung, Stadterneuerung und ,Sozialen Stadt' zur, Sicheren Stadt'

Welche Rolle spielte nun die Stadtpolitik in dem geschilderten Geschehen? Aus stadtsoziologischer Sicht sind die Vorgänge in der Großwohnsiedlung Steilshoop ein Beispiel für gelungene Stadterneuerung und Sanierung, die von sozialen Maßnahmen der Unterstützung und einer Verbesserung der Infrastruktur flankiert wurden. Dabei wurde auch auf eine Verbesserung des Sicherheitsempfindens der Bewohner hingearbeitet.

Ausgangspunkt der Entwicklung in Steilshoop ist aus städtebaulicher Sicht das räumlich konzentrierte Eingreifen des lokalen Staates auf den Wohnungsmarkt: Sozialer Wohnungsbau sollte dafür sorgen, dass allen Bewohnern der Stadt angemessener Wohnraum zu annehmbaren Bedingungen zur Verfügung steht. Aus diesem Bemühen ergaben sich in der Konsequenz die baulichräumliche Gestaltung der Siedlung sowie die von gemeinnützigen Wohnungsgesellschaften dominierte Eigentümerstruktur dieser wie vieler anderer westdeutscher Großwohnsiedlungen. Über diese Verbindung war die Stadt Hamburg später, als sich die ersten Mängel in der Struktur zeigten, in einer besonderen Verantwortung. Handlungsdruck führte zur Erklärung zum Sanierungsgebiet und anderen Maßnahmen (Einsatz von Arbeitsbeschaffungsmaßnahmen in den Einrichtungen des Stadtteils usw.), die die Quartiersentwicklung in besonderem Maße förderten.

Nun weisen die oben aufgeführten Stadtentwicklungsmaßnahmen zahlreiche Parallelen zu den heutigen Maßnahmen kommunaler Kriminalprävention in anderen bundesdeutschen Städten auf. Dies ist kein Zufall, sondern das Ergebnis ähnlicher Zielsetzungen: Sowohl in der kommunalen Kriminalprävention als auch in der sozialen Stadtentwicklung konzentrierte sich Politik mehr und mehr auf die allgemeine Verbesserung der Lebensbedingungen in bestimmten Gebieten und versuchte, neben der materiell-baulichen Struktur, zusätzlich Einfluss auf die sozialen Prozesse in den Gebieten zu nehmen. ${ }^{18}$ Allein der Modellcharakter Neu-Steilshoops wies in diese Richtung, denn hier wurden die in einem Sanierungsgebiet üblichen Maßnahmen um sozialpädagogische Handlungsweisen ergänzt.

Bereits zu Beginn der 90er Jahre zeichnete sich eine Entwicklung in der Hamburger Politik ab, die mehr noch als diese neue Form der Sanierung auf das ,Soziale' einging. Untersuchungen hatten auf die zunehmende sozial-räumlichen Polarisierung in bundesdeutschen Großstädten hingewiesen (für Hamburg siehe Alisch / Dangschat 1993). Die Konzentration benachteiligter Bevölkerungsgruppen wurde über die zunehmend ins rechte politische Feld rückenden Wahlergebnisse in Teilgebieten der Stadt zum politischen Thema (vgl. Froessler 1994: 17; Alisch / Dangschat 1994: 180; Herrmann 2001: 115). Mit der traditionellen städtebaulichen Förderung waren die komplexen Probleme in den Gebieten der ,Armen' oder ,sozial Benachteiligten' nicht zu lösen (vgl. Franke / Löhr / Sander 2000: 247f; Becker / Franke / Löhr / Schuleri-Hartje 2003: 9). Neben Mängel im Städtebau und der Infrastruktur, der hohen Umweltbelastung der Gebiete (häufig durch Verkehr oder anderen Emissionen), Problemen bzw. nicht Vorhandensein einer lokalen Ökonomie in den reinen Wohngebieten und den mangelnden Chancen der Integration der Bewohner auf dem städtischen Arbeitsmarkt sind es gewalttätig ausgetragene Konflikte (zwischen Deutschen und Migrantengruppen oder zwischen den einzelnen Gruppen untereinander) sowie ein hohes Maß an Kleinkriminalität, die den lokalen Staat auf den Plan rufen. »Kleinkriminalität ersetzt in einem durch Arbeitslosigkeit geprägten Umfeld oftmals Arbeit als materielle Basis für Konsum« (Franke / Löhr / Sander 2000: 248). Hinzu kommt die auch in der Kriminologie geäußerte Vermutung, dass die in einigen Gebieten entstehende ", abweichende Kultur' durch soziales Lernen von Kindern und Jugendlichen, die in einem Umfeld, das nur wenig Vorbilder und Repräsentanten eines ,normalen Lebens' bietet, den Sinn von Schule, Ausbildung und Beruf nicht vermittelt bekommen« (ebd.). - Wobei die sehr geringen Chancen der weniger ausgebildeten auf dem Arbeitsmarkt und die damit verbundene ,Sinnlosigkeit' von Haupt- oder manches Mal auch Realschulabschlüssen sicherlich ein Übriges tun.

Die sozialdemokratisch regierte Stadt Hamburg reagierte zum einen in Form der Modernisierung der klassischen Sanierungsvorhaben, wie oben beschrieben. Mit dem Programm, Stadtteile mit besonderem Erneuerungsbedarf' im Jahr 1993 in Nordrhein-Westfalen und dem ,Armutsbekämpfungsprogramm‘ 1994 in Hamburg übernahmen die beiden Bundesländer zudem Vorreiterfunktion in der Entwicklung einer baulich-räumliche wie soziale Aspekte umfassenden Programmatik, die politisch nicht kriminalpräventiv, sondern stadtentwicklungs- und sozialpolitisch eingeordnet wurde. ${ }^{19}$ Die ,Soziale Stadt' (und die zugrunde liegenden wissenschaftlichen Ansätze) nahm die soziale und räumliche Ausgrenzung der Bewohner in den Fokus und nicht die im Zuge der Ausgrenzung auftretende Kriminalität bzw. Gewalt und abweichendes Verhalten.
Maßnahmen und finanzielle Mittel waren nunmehr nicht mehr nur in den Sanierungsgebieten (wie Steilshoop), sondern vor allem in den Gebieten der ,sozialen Stadtentwicklung' zu konzentriert. Dabei erweiterte sich das Instrumentarium vor allem um das so genannte, Quartiersmanagement', zunächst auch häufig als, Stadtteilmanagement' bezeichnet (vgl. die Beiträge in Alisch 2001). Das über das Programm finanzierte Quartiersmanagement hatte die Aufgabe, die Mitwirkung der lokalen Akteure an der Quartiersentwicklung und Wohnumfeldgestaltung zu organisieren sowie konkrete Projektentwicklung bei den im Zusammenhang mit der Quartiersentwicklung entstehenden Projektideen $\mathrm{zu}$ betreiben. Schwerpunkte der Arbeit lagen daneben in der Moderation von sozialen Prozessen, im Konfliktmanagement, der Koordination und Vernetzung, der Öffentlichkeits- und Imagearbeit für das Gebiet sowie der Akquisition finanzieller Mittel für die Gebietsentwicklung. Es sollten sich selbst tragende Strukturen der Beteiligung und des Engagements zur Minderung der Probleme entstehen. Teilziele waren u. a. die Integration der Bewohner in die (Stadt-) Öffentlichkeit, vor allem aber auch auf dem städtischen Arbeitsmarkt (vgl. Herrmann 2001). Dies geschah durch die regelmäßige Durchführung von Bürgerversammlungen bzw. -foren, Zukunftskonferenzen und im Verlauf der Weiterentwicklung des Programms vermehrt auch in Form von Workshops mit lokalen Unternehmen sowie Schulen für eine Neuorganisation des Übergangs von der Schule in die Arbeit (vgl. Breckner / González / Herrmann 2002).

In Hamburg waren es, wie in den anderen Bundesländern auch, vor allem zwei Gebietstypen, die in die aus dieser Entwicklung der Stadterneuerung entstehenden Programme der ,Sozialen Stadt' aufgenommen wurden: Die meist am Rande der Städte gelegenen Großwohnsiedlungen sowie innenstadtnahe Bereiche, die um die vorletzte Jahrhundertwende entstanden und lange 
Zeit dem Verfall preisgegeben waren. - Steilshoop wurde nicht in dieses Programm aufgenommen, da hier auf der Grundlage der Sanierung bereits umfangreiche Maßnahmen durchgeführt worden waren und zudem eine Struktur der Kooperation vor Ort - wenn auch in anderer Form - bereits existierte (s. o.).

Zu Beginn des 21. Jahrhunderts wurde, Soziale Stadtentwicklung' schließlich bundesweit in über 200 Groß- und Mittelstädten durchgeführt. Die Bund-Länder-Initiative "Soziale Stadt « bündelte sowohl finanzielle Mittel des Bundes und der Länder in den Gebieten als auch fachliches KnowHow. Mit der Installierung so genannter ,Programmbegleitungen vor Ort' wurde zudem eine besondere Form der Evaluierung und übergeordnete Unterstützung auf den Weg gebracht, die es in Hamburg in dieser Form vorher nicht gegeben hatte. Als ein zentrales Problem in der Umsetzung der Programmatik erwies sich der Aufbau einer gebietsübergreifenden Struktur, die die Maßnahmen der sozialen Stadtentwicklung zu einem gesamtstädtischen und Fachbehörden-übergreifenden Projekt hätte zusammenfassen können (vgl. Breckner / Gonzalez / Herrmann 2003 sowie Mayer 2004: 43ff).

Gleichzeitig verstärkte sich der ,defensive Charakter $^{\prime 20}$ der kriminalpräventiven Aktivitäten, wurde durch den bereits genannten Politikwechsel im Jahr 2001 eher eine Wende zur Repression statt Prävention vollzogen. Die sozialpolitische Orientierung etwa im Umgang mit jugendlichen Straftätern wich einer expliziten Devise zur ,härteren Gangart'. Daneben waren die Bewohner von Steilshoop wie die anderer Stadtteile mit einer hohen Zahl an Arbeitslosen und ehemaligen Sozialhilfeempfängern mehr als andere von zahlreichen Kürzungsmaßnahmen im sozialen Bereich betroffen (Wegfall von Arbeitsbeschaffungsmaßnahmen, die Treffpunkte organisierten usw.). Die oben geschilderten gesamtgesellschaftlichen Entwicklungen und Veränderungen in der Organisation des Sozialstaates wirken sich in den Gebieten Benachteiligter sowohl über die höhere Betroffenheit der einzelnen Bewohner als auch über die Kürzungen in der sozialen Infrastruktur in diesem Sinne doppelt - aus. Eine umfangreiche Neuorganisation der Polizei tat ein Übriges, den Blick in die Zukunft ungewiss werden zu lassen. Längerfristige und verlässliche Planungen waren zu diesem Zeitpunkt weder im sozialpolitischen / stadtplanerischen noch im innenpolitischen Feld möglich. ${ }^{21}$ So vermitteln die Experteninterviews den Eindruck der Ungewissheit im Bezug auf den Fortgang der Dinge, sehen die Weiterentwicklung speziell in Steilshoop jedoch weiterhin positiv. Dem Netzwerk institutioneller und ehrenamtlicher Unterstützung wird Dauerhaftigkeit zugetraut. $^{22}$
Ein neues, interdisziplinäres Präventionsverständnis?

Der Blick auf die vergangene Stadtentwicklungspolitik der Stadt Hamburg und die Geschehnisse im Sanierungsgebiet Steilshoop wirft die Frage auf, ob kommunale Kriminalprävention nicht mehr als bisher geschehen auf stadtsoziologische und -planerische Erfahrungen zurückgreifen sollte, will sie dem Einfluss der baulich-räumlichen Struktur auf (Kriminalitäts-) Furcht und Ängste der Bewohner Rechnung tragen. Zukünftige Präventionspolitik müsste dann jedoch auf einen wesentlich weiter gefassten Präventionsbegriff und -verständnis aufbauen.

Ein interdisziplinäres Präventionsverständnis führt Maßnahmen und Konzepte der sozialen Stadtentwicklung (baulich-räumliche wie soziale Entwicklung in Form der Stärkung des nachbarschaftlichen Zusammenhalts oder der Förderung der Integration von Benachteiligten) mit denen der kommunalen Kriminalprävention zusammen. Den Ansätzen ist gemeinsam (vgl. in ähnlicher Weise für kriminalpräventive Ansätze Feltes 1995: 14f, Trenczek / Pfeiffer 1996: 29),

- dass Gebiete im Fokus der Politik stehen, die eine hohe Konzentration von Arbeitslosen, Sozialhilfe- bzw. Arbeitslosengeld II Empfängern aufweisen. Mit einem regionalen Ansatz wird der Blick auf "geforderte Nachbarschaften " (Herrmann 2000) gerichtet, die mit einer hohen Wohndichte, einer Vielzahl aufeinander treffender Kulturen u. v. m. zu kämpfen haben und gleichzeitig alle Merkmale aufweisen, die gemeinhin als Kriminalitätsfurcht auslösend gelten (s. o.; Kube 2003). Es ist an den vorhandenen baulich-räumlichen wie sozialen Gegebenheiten anzuknüpfen.

- dass städtebauliche und gestalterische Maßnahmen von Teilbereichen an Gebäuden und in öffentlichen Räumen mit Maßnahmen zur Nachbarschaftsförderung und Bürgerbeteiligung kombiniert werden (multifaktorieller Ansatz),

- dass Formen der Koordination und Kooperation zwischen Wohnungsgesellschaften, lokaler Polizei, mit Stellen in den Verwaltungen, Trägern der Sozial- und Jugendhilfe sowie anderen vor Ort tätigen Akteuren angestrebt werden (arbeitsteiliger und kooperativer Ansatz) sowie

- gezielt bestimmte Bewohnergruppen und Potentiale über (Partizipations-) Angebote aktiviert und in die Stadtgesellschaft integriert werden sollen (Ganzheitlicher Ansatz) ${ }^{23}$;

- schließlich wird davon ausgegangen, dass Probleme in den Gebieten sich längerfristig nur lösen lassen, wenn nicht nur die gebietsspezifischen und individuellen, sondern auch die strukturellen Ursachen angegangen werden (struktureller Ansatz).

Ziele der kommunalen Kriminalprävention sowie der sozialen Stadtentwicklung waren und sind
- die Entwicklung Politikfeld und Institutionen übergreifender Ansätze,

- die Verbesserung der Lebensqualität insbesondere in den Problemgebieten der Städte,

- eine Imageverbesserung der Städte und Stadtteile,

- die Entwicklung eines Frühwarnsystems für jeweilige Problemlagen und die Entwicklung bzw. Unterstützung der Community im Hinblick auf eine Stärkung der Potentiale, Solidarität und Zivilcourage.

Soll die eingangs erwähnte Tatsache, dass Architektur und bebaute Umwelt Einfluss auf die Sicherheit in der Stadt nehmen, ernst genommen werden, dann ist Sicherheit ein integraler Teil moderner Stadtentwicklung (vgl. Schubert 2004: 7) und Stadtentwicklung im hier verstandenen Sinne ein Bestandteil kommunaler Prävention. Sicherheit ist folgerichtig eine Querschnittsaufgabe verschiedenster Politikfelder (einer Sozial-, Verkehrs-, Wirtschafts- und Kulturpolitik ebenso wie der Kriminalpolitik; vgl. Trenczek/ Pfeiffer 1996: 14) und ist nicht mehr allein als Aufgabe der Polizei bzw. des innenpolitischen Ressorts zu sehen, sondern als ein Ergebnis von problemorientiertem, vernetzten Handeln verschiedener Akteure. Ziele der klassischen Kriminalprävention und ihr Fokus werden erweitert und ergänzt, wie die folgende Übersicht zeigt.

Ein Instrument zur Umsetzung der Maßnahmen kann dabei der Einsatz eines Quartiersmanagement sein, ${ }^{24}$ es können jedoch auch Arbeitskreise / Runde Tische der Funktionsträger des Gebiets als Koordinatoren, »Frühwarnsystem « und Entwickler von Potentialen fungieren. In Steilshoop hat es weder einen über die soziale Stadtentwicklung eingesetzten Quartiersmanager noch einen der Polizei zugehörigen expliziten Präventionsmanager gegeben. Dennoch entspricht die aufgebaute Struktur mit den Arbeitskreisen, die Beteiligung von relevanten Akteuren wie den Wohneigentümern, der Leitung des Einkaufzentrums oder auch - im Bezug auf konkrete Planungsvorhaben - der betroffenen Bewohner der Struktur, die im Rahmen der ,Sozialen Stadt' angestrebt wird und wohl auch dem ,Modernen Präventionsverständnis' (Schubert 2004) entspricht. Ein wesentlicher Bestandteil des Erfolges dieses Gremiums ist, dass die am Tisch Sitzenden gemeinsame Interessen verfolgen und über eine Entscheidungs- und Handlungsmacht verfügen, die die Beteiligten der Gremien der ,Sozialen Stadt' häufig nicht haben.

Neben dem Bezug des Geschehens zur Handlungsmacht der lokalen Entscheidungsträger braucht es auf der gesamtstädtischen Ebene im Falle einer Großstadt einer bereichsübergreifenden Organisation (vgl. im Zusammenhang mit der sozialen Stadtentwicklung Franke/Löhr/Sander 2000: 264). Die zentrale Zusammenführung der unterschiedlichen Politikfelder in Person des Ersten Bürgermeisters der Stadt hat sich im Rah- 
Erweiterung des ,klassischen' um ein interdisziplinäres Verständnis von Prävention

\begin{tabular}{|c|c|c|}
\hline & $\begin{array}{l}\text { „klassisches‘ Präventions- } \\
\text { verständnis }\end{array}$ & $\begin{array}{l}\text { interdisziplinäres Präventions- } \\
\text { verständnis }\end{array}$ \\
\hline Ziel & $\begin{array}{l}\text { Kriminalprävention durch } \\
\text { Überwachung }\end{array}$ & Verbesserung der Lebensqualität \\
\hline Fokus & Kriminalität und Täter & $\begin{array}{l}\text { Sicherheitsgefühl und geforde Nach- } \\
\text { barschaften/Benachteiligte }\end{array}$ \\
\hline Akteure & Polizei, Justiz & $\begin{array}{l}\text { Alle Akteure im und außerhalb des Gebiets, die } \\
\text { mit dem jeweiligen Problem / Vorhaben zu tun } \\
\text { haben; problemorientiertes und vernetzte } \\
\text { Handeln verschiedener Akteure (Politik un } \\
\text { Verwaltung, Institutionen und Einrichtungen, } \\
\text { wirtschaftliche und private Akteure) }\end{array}$ \\
\hline Prinzip & Kontrolle & Aktivierung \\
\hline $\begin{array}{l}\text { Umsetzung/ } \\
\text { Maßnahmen }\end{array}$ & Strafverfolgung & $\begin{array}{l}\text { Entwicklung von Netzwerken, Unterstützung } \\
\text { von Potentialen } \\
\text { Bürgerbeteiligung und Stadtumbau }\end{array}$ \\
\hline
\end{tabular}

men der sozialen Stadtentwicklung als problematisch erwiesen und bisherige Reflexionen im Hinblick auf eine erfolgreiche Präventionspolitik der Hansestadt lassen einen ähnlichen Schluss zu. Ein fächerübergreifender Landespräventionsrat hätte nicht nur die Funktion der Analyse der Situation in den einzelnen Stadtteilen, sondern wäre darüber hinaus das Gremium, in dem sich die Aktivitäten bündeln, koordinieren und an die gesamtstädtische Ebene anknüpfen ließen.

\section{Fazit}

Nach den vorangehenden Überlegungen ist ,Prävention' mehr als bisher fächer- sowie ebenenübergreifend $\mathrm{zu}$ verstehen und $\mathrm{zu}$ konzipieren. Der alleinige Blick auf kriminalpräventive Maßnahmen und hiermit verbundene Institutionen führte in der Vergangenheit dazu, dass die Hamburger Politik im innerdeutschen Ländervergleich recht schlecht abschneidet, da auf die fehlende Existenz eines Landespräventionsrates verwiesen wird. Die (präventive) Arbeit im Hamburger Sanierungsgebiet Steilshoop hat jedoch gezeigt, dass kommunale Kriminalprävention in der ehemals sozialdemokratisch regierten Stadt eher unter dem Label einer sozialpolitisch beeinflussten Stadtentwicklungspolitik als unter kriminalpräventiven Gesichtspunkten stattfand. Was in Hamburg dennoch fehlt, ist eine institutionelle Struktur und Verknüpfung der Maßnahmen in den einzelnen Gebieten zu einem gesamtstädtischen Konzept. Dieses könnte helfen, Schwierigkeiten in der konkreten Zusammenarbeit der verschiedenen Professionen vor Ort auf einer übergreifenden Ebene zu lösen, indem die Kompetenzen und Zuständigkeiten voneinander abzugrenzen sind (zu den Schwierigkeiten einer Kooperation der Professionen vgl. Kyvsgaard 1996: 146).
Das gewählte Beispiel der ,Großwohnsiedlung' verweist darauf, dass einige Dinge trotz vielerlei Bemühungen nicht zu ändern sind. Das für Viele unattraktive Erscheinungsbild einer Großwohnsiedlung lässt sich nur bedingt beeinflussen: Aus der Großwohnsiedlung wird nie ein Jugendstilquartier, wie es in Teilen in den innenstadtnahen Gründerzeitvierteln der Benachteiligten zu beobachten ist. Verbesserungen des Wohnumfeldes können die Attraktivität des Stadtteils, auch dies zeigte das Beispiel, jedoch erhöhen. Auch ist das nach wie vor schlechte Image des Stadtteils in den Hintergrund getreten, wird aber bei unseren Befragungen auf Nachfrage ,reproduziert'. Die Hartnäckigkeit, mit der sich die alten Geschichten halten, liegt zum Teil an der aus heutiger Sicht weiterhin problematischen Baustruktur. Eine andere Ursache liegt darin, dass nur die Bewohner oder hier Arbeitende einen Anlass haben, die Siedlung überhaupt zu betreten. Den Bewohnern anderer Stadtteile sowie vielen Vertretern der Politik und Verwaltung bleiben die Veränderungen weitgehend verborgen, da sie weniger als die vorherige Dramatik über die Medien vermittelt werden.

Andere Ursachen für Trends in der Gebietsentwicklung sind eher auf gesamtgesellschaftlicher Ebene zu suchen: Das Wegbrechen des Einzelhandels in vielen Gebieten und seine im Vergleich zu großen ,Konsumwelten' sinkende Attraktivität sind Teilelemente gesamtstädtischer Entwicklungen der Konzentration. Sie lässt sich lokal nur begrenzt aufhalten. Auch sind wohnortnahe Arbeitsplätzen und lokale Ökonomie in einer Monostruktur des Wohnens schwerer zu schaffen, als in einem Altstadtquartier (Stichwort: Nutzungsmischung). Die vorhandenen Räume können jedoch so gestaltet werden, dass sich möglichst viele Bewohnergruppen an Orten der Kommunikation und der zufälligen Begegnung angstfrei aufhalten können. In Steilshoop wurde diesbezüglich in der Vergangenheit viel erreicht: So sprechen die Bewohner lediglich von einem Unwohlsein, dass sie manchmal in der durch die Jugendlichen dominierten Mittelachse der Siedlung empfinden. Dennoch bleiben, leere Fenster in den Einzelhandelszentren ein Problem, dem sich auch die städtische Politik stellen muss.

Insgesamt zeigt sich, dass für die Entwicklung konkreter Handlungsansätze ein genaues Hinsehen erforderlich ist: Baulich-räumliche Strukturen sind ebenso wie Interessen und Funktionen der vor Ort tätigen Akteure einzubeziehen. Hinzu kommen die wirksamen (Handlungs-) Traditionen und Geschichten sowie die sich nicht verbal artikulierenden Menschen, die mit ihren Werten und Lebensstilen die Gebietsentwicklung prägen. Dieses widerspricht der Wirksamkeit einer allgemein, im ganzen Europa und in jeder baulich-räumlichen sowie sozialen Struktur gültigen Norm (s. o.). Der ,Modellversuch Steilshoop', der in seiner Planung den Prototyp einer lebenswerten Großwohnsiedlung darstellen sollte, zeigt die Grenzen allgemeingültiger Vorgaben auf. Sie kann, auch im Bezug auf die baulich-räumlichen Aspekte, lediglich eine erste Orientierung bieten, denn der Raum gewinnt letztlich erst durch das Soziale, d. h. die dort lebenden Menschen, seine endgültige Form und Lebendigkeit. Auch hat sich die Erkenntnis noch nicht durchgesetzt, dass nicht ein punktuelles Eingreifen in Gebiete mit Handlungsbedarf, sondern ein stetiges Begleiten der sich immer wieder neu gestaltenden Probleme von Nöten ist. Steilshoop war und ist auch hierfür ein Beispiel. Bei allen Anstrengungen vor Ort brauchen die Akteure eine stetige, sich den jeweiligen gesellschaftlichen Rahmenbedingungen stellende Unterstützung der politischen und öffentlichen Akteursgruppen.

Dr. Heike Herrmann war Hamburger Projektverantwortliche in dem durch die Europäische Union geförderten Projekt »Insecurities in European Cities «. Sie lehrt an der Universität Bielefeld.

\section{Literatur}

Alisch, Monika (2001): Stadtteilmanagement. Voraussetzungen und Chancen für die Soziale Stadt. 2. Aufl. (Orig. 1998). Opladen: Leske + Budrich.

Alisch, Monika \& Dangschat, Jens (1993): Die solidarische Stadt - Ursachen von Armut und Strategien für einen sozialen Ausgleich. Darmstadt: Verl. für wiss. Publikationen

Alisch, Monika \& Dangschat, Jens (1994): Ziele und Strukturen der Stadtentwicklung des Sozialen Ausgleichs in Hamburg. In: Froessler, Rolf \& Lang, Markus \& Selle, Klaus \& Staubach, Rainer (Hg.): Lokale Partnerschaften: Die Erneuerung benachteiligter Quartiere in Europäischen Städten. Basel, Boston, Berlin. S. 176-189.

Baisch-Weber, Anja (2000): Sozialraumanalyse Wandsbek. Region II. Bramfeld, Steilshoop, Alstertal. Jugendhilfeplanung Hamburg-Wandsbek. Ein Entwurf. Bannenberg, Britta \& Coester, Marc \& Marks, Erich (Hg.) (2005): Kommunale Kriminalprävention. Ausgewählte Beiträge des 9. Deutschen Präventionstages. 17. und 18. Mai 2004 in Stuttgart. Godesberg: Forum Verlag. 
Becker, Heidede \& Franke, Thomas \& Löhr, Rolf-Peter \& Schuleri-Hartje, Ulla-Kristina (2003): Das Programm Soziale Stadt: Von der traditionellen Stadterneuerung zur integrativen Stadtteilentwicklung. In: Deutsches Institut für Urbanistik (Hg.): Strategien für die Soziale Stadt. Erfahrungen und Perspektiven - Umsetzung des Bund-Länder-Programms »Stadtteile mit besonderem Entwicklungsbedarf - die soziale Stadt «. Berlin.

Beyerlein, Bernd (1980): Erklärung der Fluktuation der Mieter im Wohnmodell Steilshoop unter besonderer Berücksichtigung des sozialräumlichen Standortes. Unveröffentlichte Diplomarbeit im Bereich Stadtsoziologie der Universität Hamburg.

Breckner / González / Herrmann (2002): Endbericht der »Programmbegleitung vor Ort « des Modellgebiets Hamburg-Altona-Lurup im Rahmen des Bund-Länder-Programms »Soziale Stadt. Hamburg.

Brüsemeister, Thomas (2000): Qualitative Forschung. Ein Überblick. Wiesbaden: Westdeutscher Verlag.

Flade, Antje (2003): Die sichere Stadt. In: Kerner, HansJürgen \& Marks, Erich (Hg.): Internetdokumentation Deutscher Präventionstag. Hannover.

http://www.praeventionstag.de/content/2_praev/ doku/flade/index 2 flade.html

Flick, Uwe (1995): Qualitative Forschung. Theorie, Methoden, Anwendung in Psychologie und Sozialwissenschaften. Reinbek bei Hamburg: Rowohlt

Franke, Thomas \& Löhr, Rolf-Peter \& Sander, Robert (2000): Soziale Stadt - Stadterneuerungspolitik als Stadtpolitikerneuerung. In: Archiv für Kommunalwissenschaften. 39. Jahrg. 2000. II. Halbjahresband. Stuttgart. S. 243-268.

Froessler, Rolf (1994): Integrierende Politik: Aufgaben, Inhalte und Formen staatlicher Programme zur Erneuerung benachteiligter Quartiere. In: Froessler, Rolf \& Lang, Markus \& Selle, Klaus \& Staubach, Rainer (Hg.): Lokale Partnerschaften: Die Erneuerung benachteiligter Quartiere in Europäischen Städten. Basel, Boston, Berlin. S. 8-35.

Garland, David (2001): The Culture of Control. Crime and Social Order in Contemporary Society. Oxford: University Press.

Häußermann, Hartmut (2000): Die Krise der «Sozialen Stadt«. In: Aus Politik und Zeitgeschichte. Beilage zur Zeitschrift »Das Parlament «. Heft B 10-1. Bonn.

Harms, Hans \& Schubert, Dirk (1988): Gross-Siedlungen in Hamburg - Übersicht, Bestandsaufnahme, Probleme. 2.Auflage. Technische Universität Hamburg-Harburg.

Heinz, Wolfgang (2005): Kommunale Kriminalprävention aus wissenschaftlicher Sicht. In: Bannenberg, Britta \& Coester, Marc \& Marks, Erich (Hg.): Kommunale Kriminalprävention. Ausgewählte Beiträge des 9. Deutschen Präventionstages. 17. und 18. Mai 2004 in Stuttgart. Godesberg: Forum Verlag. S. 9-30.

Hermann, Dieter \& Laue, Christian (2001): Ökologie und Lebensstil - Empirische Analysen zum »brocken windows «- Paradigma. In: Jehle, Martin (Hg.): Raum und Kriminalität: Sicherheit in der Stadt. Mönchengladbach: Godesberg. S. 89-120.

Dies. (2004): Wirkungen kommunaler Kriminalprävention - Ein Fallbeispiel. In: Bannenberg, Britta \& Coester, Marc \& Marks, Erich (Hg.) (2005): Kommunale Kriminalprävention. Ausgewählte Beiträge des 9 . Deutschen Präventionstages. 17. und 18. Mai 2004 in Stuttgart. Godesberg: Forum Verlag. S. 197-208

Herrmann, Heike (2000): Sozialraum Quartier. Konfliktfelder und Perspektiven in Großstadtregionen. In: Journal für Konflikt- und Gewaltforschung, Heft 2/2000. Universität Bielefeld. S. 207-223.

Herrmann, Heike (2001): Bürgerforen. Ein lokalpolitisches Experiment der Sozialen Stadt. Opladen: Leske + Budrich.

Herrmann, Heike \& Sessar, Klaus \& Weinrich, Martin (2002): Unsicherheiten in der Moderne am Beispie der Großstadt. Kontexte eines europäischen Forschungsprojekts. In: Hanak, Gerhard \& Stangl, Wolfgang (Hg.): Jahrbuch für Rechts- und Kriminalsoziologie 2002. Innere Sicherheiten. Baden-Baden: Nomos. S 251-286.

Hess, H. (1999): Fixing Broken Windows and Bringing Down Crime. In: Kritische Justiz, 1/1999, S. 32ff.
Kastner, Peter \& Sessar, Klaus (Hg.)(2001): Strategien gegen die anwachsende Jugendkriminalität und ihre gesellschaftlichen Ursachen. Berichte der EnqueteKommission der Hamburger Bürgerschaft. Eine Dokumentation. Hamburger Studien zur Kriminologie und Kriminalpolitik Band 27. Münster: LIT.

Kodolitisch, Paul von (2003): Einführung: Sicherheit in der Stadt. In: Deutsche Zeitschrift für Kommunalwissenschaften. 42. Jahrgang. 2003/I. S. 5-10.

Kosak, Egbert (1994): Hamburg. Stadt am Fluss. Herausgegeben von der Baubehörde der Freien und Hansestadt Hamburg. Hamburg: Ellert \& Richter Verlag.

Kube, Edwin (2003): Städtebau und Kriminalität. In: Sicherheit und Kriminalität. Zeitschrift »Bürger im Staat «, Heft 1/2003.

Kyvsgaard, Britta (1996): Kommunale Kriminalprävention - Erfahrungen aus Dänemark. In: Trenczek, Thomas \& Pfeiffer, Harmut (Hg.): Kommunale Kriminalprävention - Paradigmenwechsel und Wiederentdeckung alter Weisheiten. Bonn/ Mönchengladbach Forum Verlag. S. 141-153.

Löhr, Rolf (2001): Soziale Stadt - sichere Stadt. In: Zeitschrift des Deutschen Forums für Kriminalprävention. Heft 1/2001. S. 23.

Mayer, Hans-Norbert (2004): Hamburgisches Stadtteilentwicklungsprogramm - Zwischenevaluation 2003 in acht Quartieren. Gutachten der Arbeitsgruppen Stadtforschung der Universität Oldenburg im Auftrag der Behörde für Bau und Verkehr der Hansestadt Hamburg. Hamburg.

Meier, Uwe (1985): Kriminalität in Neubausiedlungen: das Beispiel Hamburg Steilshoop. Frankfurt/M., Bern, New York: Peter Lang.

Oberwittler, Dietrich (2003): Die Entwicklung von Kriminalität und Kriminalitätsfurcht in Deutschland Konsequenzen für die Kriminalitätsprävention. In Deutsche Zeitschrift für Kommunalwissenschaften. 42. Jahrgang. 2003/I. S. 31-53.

Papendorf, Knuth \& Neth, Axel (1996): Praxiserfahrungen bei der Erstellung einer Kriminologischen Regionalanalyse und der Initiierung eines »Kriminalpräventiven Rates « - das Beispiel Lübeck. In: Trenczek, Thomas \& Pfeiffer, Harmut (Hg.): Kommunale Kriminalprävention - Paradigmenwechsel und Wiederentdeckung alter Weisheiten. Bonn/Mönchengladbach: Forum Verlag. S. 104-117.

Schubert, Herbert (2001): Einleitung. In: Niedersächsisches Ministerium für Soziales, Frauen, Familie und Gesundheit (Hg.): Sicherheit planen und gestalten. Realisierung der städtebaulichen und wohnungswirtschaftlichen Kriminalprävention durch Leitbilder und Verfahren. Veröffentlichung des Landes Niedersachsen. S. 6-14.

Schubert, Herbert \& Schnittger, Angela (2002): Sicheres Wohnquartier - gute Nachbarschaft. Handreichung zur Förderung von Kriminalprävention im Städtebau und in der Wohnungsbewirtschaftung. Erarbeitet im Auftrag des Niedersächsischen Innenministeriums. Hannover.

Schwind, Hans-Dieter (2001): Kriminalitätsfurcht als kommunales Problem. In: Zeitschrift des Deutschen Forums für Kriminalprävention. Heft 1/2001. S. 20f

Sessar, Klaus (1984): Jugendstrafrechtliche Konsequenzen aus jugendkriminologischer Sicht: Zur Trias von Ubiquität, Nichtregistrierung und Spontanbewährung im Bereich der Jugendkriminalität. In: Walter, M. \& Koop, G. (Hg.): Die Einstellung des Strafverfahrens im Jugendrecht. Chancen und Risiken eines neuen kriminalpolitischen Weges sowie Erfahrungen und Anregungen aus der Praxis. Kriminalpädagogische Praxis, 5. Vechta, S.26-50.

Sessar, Klaus \& Herrmann, Heike \& Keller, Wolfgang \& Weinrich, Martin \& Breckner, Ingrid (2003): Local Report Hamburg. Unveröffentlichtes Manuskript des Lokalen Berichts im Rahmen des Projekts »Insecurities in European Cities. Crime-Related Fear Within the Context of New Anxieties and Community-Based Crime Prevention. Hamburg.

Skogan, Wesley (1990): Disorder and Decline. Crime and the Spiral of Decay in American Neighborhoods. New York: Free Press.
Soomeren van, Paul \& Mölk, Julia (2001) Europäische Norm ENV 14383-2. In: Niedersächsisches Ministerium für Soziales, Frauen, Familie und Gesundheit (Hg.): Sicherheit planen und gestalten. Realisierung der städtebaulichen und wohnungswirtschaftlichen Kriminalprävention durch Leitbilder und Verfahren. Veröffentlichung des Landes Niedersachsen. S. 37-42.

Stadtentwicklungsbehörde der Freien und Hansestadt Hamburg (FHH) (Hg.) (1994): Armutsbekämpfung in Hamburg. Zusätzliche Maßnahmen gegen Armut als Bestandteil sozialer Stadtentwicklung. Hamburg.

Stangl, Wolfgang (2005): Viel Sicherheit durch wenig Kriminalprävention? Bemerkungen zum »Fall Wien«. In: Neue Kriminalpolitik. Forum für Praxis, Politik und Wissenschaft. 17. Jahrgang. S.9-12.

Stock, Jürgen (2001): Kriminalprävention in Deutschland. Eine Strukturskizze. In: Zeitschrift des Deutschen Forums für Kriminalprävention. Heft 1/2001. S. 12-14.

Weicht, Christian (2003): Kriminalprävention durch Umweltgestaltung - Europäische Erfahrungen in Städtebau und Architektur. In: Kerner, Hans-Jürgen \& Marks, Erich (Hg.): Internetdokumentation Deutscher Präventionstag. Hannover.

http://www.praeventionstag.de/content/8_praev/ doku/weicht/index 8 weicht.htm

Ziercke, Jörg (2001): Nutzen und Mehrwert für die Kriminalprävention in Deutschland. In: Zeitschrift des Deutschen Forums für Kriminalprävention. Heft $1 / 2001$. S. 2-6

\section{Fußnoten}

1 Dies heißt selbstverständlich nicht, dass nichts existiert. Insbesondere die fundierten Regionalanalysen des Landeskriminalamtes Hamburg böten eine gute Grundlage für erste Konzepte. Auch existieren in Hamburg-Harburg sowie - Altona zwei Sicherheitskonferenzen in der Stadt, die die Belange der Bürgerinnen und Bürger in den Gebieten aufgreifen.

2 Als so genannter Stadtstaat ist die Freie und Hansestadt Hamburg gleichzeitig eines der 16 deutschen Bundesländer und besitzt neben einem Landesparlament (Bürgerschaft) und einer Landesregierung (Senat) und Landesministerien (Behörden) eine alle Gerichtszweige umfassende Justiz. Das Polizeirecht ist grundsätzlich länderrechtlich geregelt, das heißt Hamburg verfügt über eine eigene Polizeihoheit.

3 Das Projekt stand unter der Leitung von Prof. Klaus Sessar und Martin Weinrich, Universität Hamburg.

4 Die Experten waren Angehörige der Polizei, der Verwaltung, des mit der Sanierung beauftragten Unternehmens, der Wohnungsgesellschaften, sozialer Einrichtungen, Medienvertreter und andere in den Gebieten Tätige.

5 Zum folgenden vgl. Teil B, Kap. 7.2 des Abschlussberichts (Sessar / Herrmann / Keller / Weinrich / Breckner 2003).

6 Ein weiterer Bereich ergibt sich aus dem Handlungsbedarf bezüglich der Gefahrenabwehr und der Verfolgung des Terrorismus. Auf diesen Bereich kann aufgrund seiner Besonderheit an dieser Stelle nicht näher eingegangen werden.

7 Die statistischen Zahlen beziehen sich ausnahmslos auf den Stadtteil ,Steilshoop' und stammen aus der im Internet zur Verfügung stehenden Materials des Gemeinsamen Statistischen Landesamtes Hamburgs und Schleswig-Holsteins'. Der Stadtteil umfasst geringfügig mehr Wohneinheiten als die eigentliche Großwohnsiedlung, die am Ende der 60er und beginnenden 70er Jahre gebaut wurde.

8 Die mit ,EX' gekennzeichneten Verweise beziehen sich auf die in Hamburg durchgeführten Experteninterviews.

9 Meier weist darauf hin, dass der generelle Blick in die Kriminalstatistik keine hohen Kriminalitätsraten des Stadtteils ausweist (vgl. Meier 1985: 254f); eine räum- 
lich differenzierte Betrachtung verdeutlicht jedoch die Konzentration von Kriminalität und sozialen Problemen in den Blöcken der Mittelachse (ebd.: 327). Die hier herrschende Atmosphäre strahlte mit hoher Wahrscheinlichkeit auf die ganze Siedlung aus.

10 In den 80er Jahren stellten Studien fest, dass insbesondere männliche Jugendliche entwicklungsbedingt hin und wieder kleinere Delikte begehen, nur selten erwischt werden und im Allgemeinen von alleine wieder aufhören, sowie sie älter geworden sind (vgl. Sessar 1984).

11 Die hohe Wohndichte bewirkt »dass Menschen mehr Zeit außerhalb ihrer zu kleinen Wohnungen verbringen, ihre Kinder weniger überwachen und das Konfliktpotential innerhalb der Familien höher ist « (Hermann / Laue 2001: 100).

12 Die Bezeichnung ,Sanierung‘ wird im Folgenden beibehalten, obwohl die (sich über einen Zeitraum von 10 Jahren und länger erstreckenden) Maßnahmen zu Programmen der Sanierung, Revitalisierung und ,Stadterneuerung in kleinen Schritten' (SteP) gehörten.

13 Vgl. zur Einbettung der Zahlen in die gesamtstädtische Entwicklung Sessar/Herrmann/Keller/Weinrich / Breckner 2003.

14 Der seit den 90er Jahren im Verlauf der ökonomischen Krise einsetzende Rückzug des Sozialstaates aus verschiedensten Leistungen bedeutete auch einen
Rückzug aus dem sozialen Wohnungsbau. Es wurden kaum bis keine neuen Bestände geschaffen und ehemalige Sozialwohnungen aus den Mietpreis-Bindungen genommen oder privatisiert, so dass weitere Investitionen möglich wurden und die Mieten stiegen. Diese Liberalisierung des Wohnungsmarktes führte zu einer weiteren Konzentration des Bestandes in den Gebieten, in denen dieser Mechanismus nicht einsetzte. 15 Statistiken hierzu liegen noch nicht vor.

16 Die Ergebnisse beziehen sich zum überwiegenden Teil auf die Großwohnsiedlung; ein kleiner Teil der Befragten sind Bewohner eines angrenzenden Stadtteils sowie ,Alt-Steislhooper', da das die Grenzen des Untersuchungsgebiets über die eigentliche Großwohnsiedlung hinaus gingen.

17 Bereits im Dezember 2002 titelte die Zeitung die WELT: "Geldmangel: Sozialprojekten in Steilshoop droht das Aus « (20.12.2002).

18 Das durch die damalige Stadtentwicklungsbehörde herausgegebene, sich $u$. a. auf das Sanierungsgebiet Steilshoop beziehende Konzept ,Stadterneuerung Hamburg' (1996) ließt sich heute wie ein Vorläufer der Sozialen Stadtentwicklung (s. u.).

19 Das Hamburger Programm wurde später als ,Programm sozialer Stadtentwicklung' weitergeführt (vgl. hierzu den Bericht von Mayer 2004).
20 So auch die Charakterisierung von Stangl für Wien (2005: 11).

21 So wurde etwa auch die Stadtentwicklungsbehörde als Fachbehörde (vergleichbar den Ministerien anderer Bundesländer) aufgelöst und die Zuständigkeit der Fachbehördenebene insgesamt neu sortiert.

22 Dieser Optimismus ließe sich in Richtung einer bestehenden, von vielen Schultern gestützten »culture of control« (Garland 2001) interpretieren, die im Großen und Ganzen den politischen und gesellschaftlichen Veränderungen in der Stadt standhält. Die Zukunft wird erst zeigen, ob die Sparmaßnahmen in den verschiedensten Bereichen nicht doch zu einem erneuten Umkippen der (für die Enge und Struktur der Großwohnsiedlung erstaunlich) friedlichen Situation führen können.

23 Unter dem ,ganzheitlichen Ansatz' verstehen Feltes und Trenczek / Pfeiffer dagegen die umfassende Sicht auf die Probleme.

24 Der Ministerialdirigent Jörg Ziercke (Leiter der Polizeiabteilung im Innenministerium Schleswig-Holstein) schlägt die Entwicklung eines Berufsbildes des ,kommunalen Präventionsmanagers' vor (Ziercke 2001: 4).

\section{Wie oft ängstigen sich Ängstliche, wenn sie sich ängstigen? Häufigkeit und Intensität von Krimina- litätsängsten am Beispiel der Wiener Bevölkerung}

Gert Feistritzer und Wolfgang Stangl

\begin{abstract}
- mpirische Studien zu Fragen der Kriminali-
- tätsängste nationaler oder städtischer Bevölkerungen gehören $\mathrm{zu}$ den häufigsten Themen, mit denen sich die Kriminalsoziologie in den letzten rund 20 Jahren in Europa und den USA beschäftigt hat. Vielfach verbinden sich derartige Studien mit Fragen nach Möglichkeiten, wie künftig mit Kriminalitätsängsten umzugehen sei, oder auf welche Weise man sie verhindern könne, womit das zweite große kriminalsoziologische Forschungsthema angesprochen ist: Die Kriminalprävention in ihren verschiedenen ideologischen und thematischen Bezügen.
\end{abstract}

Die Zusammenführung beider Themen auf der Ebene der jeweils nationalen, aber auch der europäischen Justiz- und Innenpolitik führte zur Herausbildung des kriminalpolitischen "Superthemas «, dem deswegen so große Bedeutung zukommt, weil es aus dem engeren "Sachbereich « herausgetreten ist und sich an viele andere Sachthemen angelagert hat: Wir meinen das Thema der »Inneren Sicherheit « (vgl. Stangl 2003). Es ist dies mittlerweile ein politisches, ökonomisches wie auch wissenschaftliches Feld, auf dem die unterschiedlichsten Interessengemeinschaften, Perspektiven und Akteure ihren Platz finden und

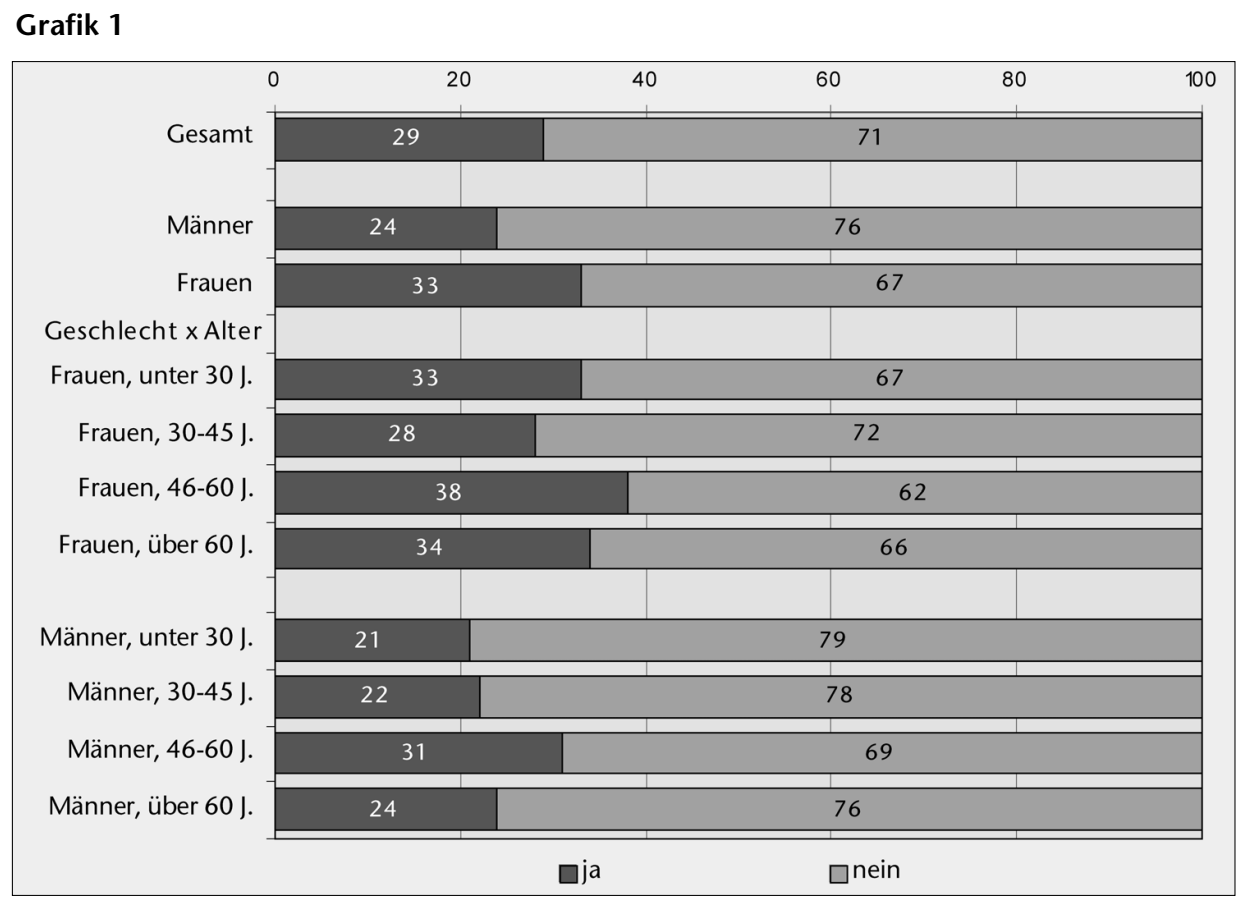

Frage: Haben Sie im letzten Jahr einmal Angst gehabt, in Wien Opfer einer Straftat zu werden? (in Prozent) 